\title{
Polymorphisms of genes in nitric oxide-forming pathway associated with ischemic stroke in Chinese Han population
}

\author{
Jiang-tao YAN ${ }^{1, \#}$, Lan ZHANG ${ }^{1, \#}$, Yu-jun XU ${ }^{1}$, Xiao-jing WANG ${ }^{2}$, Cong-yi WANG ${ }^{3}$, Dao-wen WANG ${ }^{1, *}$ \\ ${ }^{1}$ Institute of Hypertension and Department of Internal Medicine, Tongji Hospital, Tongji Medical College, Huazhong University of Sci- \\ ence and Technology, Wuhan 430030, China; ${ }^{2}$ Department of Oral Biology, School of Dental Medicine, University of Pittsburgh, Pitts- \\ burgh, PA 15219, USA; ${ }^{3}$ Center for Biotechnology and Genomic Medicine, Medical College of Georgia, Augusta, GA 30912, USA
}

Aim: To investigate the association of polymorphisms in four critical genes implicated in the NO-forming pathway with ischemic stroke (IS) in a Chinese Han population.

Methods: DNA samples of 558 IS patients and 557 healthy controls from Chinese Han population were genotyped using the Taqman ${ }^{\mathrm{TM}}$ $7900 H T$ Sequence Detection System. Six SNPs (rs841, rs1049255, rs2297518, rs1799983, rs2020744, rs4673) of the 4 related genes (eNOS, iNOS, GCH1, and CYBA) in the NO forming pathway were analyzed using the SPSS 13.0 software package for Windows. Results: One SNP located in the intron of GCH1 (rs841) was associated with IS independent of the traditional cardiovascular risk factors in co-dominant and dominant models $(P=0.003, q=0.027 ; P=0.00006, q=0.0108$; respectively). Moreover, the combination of rs1049255 CC+CT and rs841 GA+AA genotypes was associated with significantly higher risk for IS after adjustments (OR=1.73, 95\% Cl: 1.27-2.35, $P<0.0001, q<0.0001)$.

Conclusion: The data suggest that genetic variants within the NO-forming pathway alter susceptibility to IS in Chinese Han population. Replication of the present results in other independent cohorts is warranted.

Keywords: ischemic stroke; nitric oxide; polymorphism; genetics; Chinese Han population

Acta Pharmacologica Sinica (2011) 32: 1357-1363; doi: 10.1038/aps.2011.114; published online 3 Oct 2011

\section{Introduction}

Ischemic stroke (IS), known to be a multifactorial disorder, is the leading cause of disability and the second leading cause of death in China ${ }^{[1]}$. IS usually results from hypertension, atherosclerosis, diabetes, smoking, vasculitis or other etiologies. Beyond the conventional risk factors, evidence is accumulating that genetic factors may also contribute to the risk of stroke development ${ }^{[2]}$.

Nitric oxide (NO) is synthesized by the enzyme nitric oxide synthase (NOS) from $L$-arginine and oxygen in endothelial cells, neurons, glia and macrophages ${ }^{[3]}$. NO plays an important role in the control of cerebral blood flow, thrombogenesis, and the modulation of neuronal activity ${ }^{[4]}$. High concentrations of NO originating from cerebral ischemia mediate

\footnotetext{
\# These authors contributed to this work equally.

* To whom correspondence should be addressed.

E-mail dwwang@tjh.tjmu.edu.cn

Received 2010-11-11 Accepted 2011-07-17
}

inflammatory and cytotoxic pathways leading to neuronal death $^{[5]}$. NO is also important to protect vessels against atherosclerosis $^{[6]}$. NO bioavailability is tightly regulated by a balance between its production and detoxification or degradation, and therefore, cerebral ischemia could be related to abnormalities in the expression and activity of NOS.

Several potential functional polymorphisms in the nitric oxide-forming pathway have recently been discovered, including (1) Leu608Ser (rs2297518) in inducible $[i N O S]^{[7]},(2,3)$ Glu298Asp (rs1799983) and T-786C (rs2070744) in the promoter region of endothelial $[e N O S]^{[8-12]},(4,5)$ Tyr72His (rs4673) and $\mathrm{C}+640 \mathrm{~T}$ (rs1049255) in the $3^{\prime}$-untranslated region (UTR) of the cytochrome b-245, alpha polypeptide gene $(C Y B A)^{[13-16]}$, which encodes the p22phox subunit of the NADPH oxidase, and (6) G+243A (rs841) in the 3'-UTR of the GTP cyclohydrolase 1 gene $(\mathrm{GCH} 1)^{[17]}$. With special attention to the biological process of cerebral ischemia regulation, we investigated whether polymorphisms in these genes implicated in the pathway of NO formation are associated with IS in a large cohort in the Chinese Han population. 


\section{Materials and methods}

\section{Study population and data collection}

This was a multicenter, collaborative study for assessment of risk factors for stroke sponsored by the Ministry of Science and Technology of China. The study protocol was approved by the review board of Tongji Medical College at Huazhong University of Science and Technology and the ethics committees at all participating hospitals. An informed consent form was obtained from all participants.

A total of 558 IS patients were recruited between November 2004 and June 2006 from five hospitals in Wuhan, China. Only 2 subtypes of stroke - cerebral thrombosis (atherothrombosis) and lacunar infarction (lacunar) - were included. Subjects with subarachnoid hemorrhage, embolic brain infarction, brain tumors and cerebrovascular malformation were excluded from the study, as were those with severe systemic diseases such as pulmonary fibrosis, endocrine and metabolic disease (except diabetes mellitus), severe inflammatory diseases, autoimmune disease, tumors and serious chronic diseases (eg, hepatic cirrhosis and renal failure). Subjects with cardioembolic stroke and documented atrial fibrillation were also excluded from our study. Stroke diagnosis was based on the results of neurological examination and CT or MRI according to the International Classification of Diseases, ninth edition. Five hundred fiftyseven ethnically and geographically matched controls were randomly selected either from normal individuals of nearby community-based residents $(89.6 \%)$ or inpatients $(10.4 \%)$ with minor illnesses. All control subjects were free of neurological diseases following the same exclusion criteria as cases. They were also asked for a detailed medical history and received a physical examination of neurological systems, including an evaluation of body mass index.

\section{DNA isolation and genotyping}

DNA was extracted from leukocytes as previously described ${ }^{[18]}$. All samples were genotyped using the Taqman ${ }^{\mathrm{TM}} 7900 \mathrm{HT}$ Sequence Detection System according to the manufacturer's instructions. Each assay was conducted using $10 \mathrm{ng}$ DNA in a $5 \mu \mathrm{L}$ reaction consisting of TaqMan ${ }^{\mathrm{TM}}$ universal PCR master mix (Applied Biosystems, Foster City, CA, USA), forward and reverse primers and FAM and VIC labeled probes designed by Applied Biosystems [ABI Assays-on-Demand (rs841, C_9866639_10; rs1049255, C_7516913_10; rs2297518, C_11889257_10; rs1799983, C_3219460_20; rs4673, C_2038_20) and Assays-on-Design (rs2070744)]. Allelic discrimination was measured automatically using the Sequence Detection Systems 2.1 software (autocaller confidence level 95\%). A total of $10 \%$ of all genotypes were repeated in independent PCRs to check for consistency and to ensure intraplate and interplate genotype quality control. No genotyping discrepancies were detected between the repeated samples. In addition, all the DNA samples for cases and controls were run in the same batches.

\section{Statistical analysis}

Statistical analysis were performed with the SPSS 13.0 software package for Windows (SPSS Inc, Chicago, IL, USA). The normality of quantitative variable distribution was assessed using the 1-sample Kolmogorov-Smirnov test, and a transformation was applied to non-normal variables when necessary. Summary statistics were expressed as the mean \pm standard error or as percentages. The $\chi^{2}$ test was used to assess the deviation from Hardy-Weinberg equilibrium for genotype frequencies in both cases and controls. Continuous variables were compared between cases of stroke and controls using Student's $t$-test. Frequencies of categorical variables were compared by $x^{2}$ test or Fisher's exact test. The potential independent role of each single-nucleotide polymorphism (SNP) on stroke was investigated with multiple unconditional logistic regression analysis adjusted for age, sex, body mass index, hypertension, hyperlipidemia, diabetes mellitus and smoking status. To minimize the false positive results generated from the multiple statistical tests used in our analysis, we adopted a method proposed by Story and Tibshirani to estimate the FDR (false discovery rate)based $q$ value using QVALUE software (setting [lambda] $=0$, false discovery rate level $<0.05)^{[19]}$. All association analyses were conducted in three genetic models: co-dominant, dominant and recessive. Power calculations were performed using the QUANTO software program ${ }^{[20]}$ (Version 1.2.3).

\section{Results}

\section{Baseline characteristics of the subjects}

Table 1 summarizes the clinical characteristics of individuals enrolled in the study. The mean age, gender ratio and total cholesterol level were similar in cases and controls. Expectedly, there were significantly higher percentages of hypertension, diabetes mellitus, hyperlipidemia, and smoking in overall IS and subtype groups versus the controls $(P<0.05)$. Body mass index, systolic blood pressure, and diastolic blood pressure were also higher in cases than controls. Compared to the control group, patients in both the overall IS and subtype groups had significantly lower HDL cholesterol $(P<0.05)$.

\section{Genotypes in relation to ischemic stroke and its subtypes}

Next, we assessed associations between six SNPs from four related genes (three at two isoforms of NOS, one at GCH1, and two at CYBA) and IS in the Chinese Han population. All genotype distributions were consistent with Hardy-Weinberg equilibrium $(P>0.05)$. Table 2 lists single SNP allelic frequencies of the four genes among IS subjects $(n=558)$ and control subjects $(n=557)$. Mutiplicative-type corrections such as Bonferroni corrections for correlated genetic factors and tests are highly conservative. Therefore, we present the $q$ value, a measure of false discovery rate expected for a given $P$ value in the follow-up analysis. Notably, rs 841 in the intron region of GCH1 demonstrated allelic frequency differences between overall IS and lacunar stroke subjects compared with controls ( $P=0.001$ and 0.002 , respectively), which maintained statistical significance after multiple comparison correction $(q=0.018$ for both) (Table 2).

To further investigate how each of the SNP alleles interact in conferring genetic risk for IS, we conducted a genotypic 
Table 1. Baseline characteristics of patients.

\begin{tabular}{|c|c|c|c|c|}
\hline Variable & Control & Ischemic stroke & Atherothrombosis & Lacunar infarction \\
\hline$n$ & 557 & 558 & 410 & 148 \\
\hline Age, year & $62.2 \pm 9.3$ & $61.0 \pm 9.8$ & $60.0 \pm 10.0^{b}$ & $64.1 \pm 8.2^{b}$ \\
\hline Men, \% & 62.1 & 64.7 & 65.4 & 62.8 \\
\hline $\mathrm{BMI}, \mathrm{kg} / \mathrm{m}^{2}$ & $23.7 \pm 3.2$ & $24.5 \pm 3.7$ & $24.4 \pm 3.4$ & $24.7 \pm 4.4$ \\
\hline $\mathrm{SBP}, \mathrm{mmHg}$ & $131.3 \pm 20.8$ & $146.6 \pm 23.3^{b}$ & $147.1 \pm 24^{b}$ & $144.9 \pm 20.9^{b}$ \\
\hline DBP, mmHg & $78.8 \pm 11.1$ & $86.4 \pm 13.9^{b}$ & $87 \pm 14.2^{b}$ & $84.9 \pm 12.9^{b}$ \\
\hline $\mathrm{TCH}, \mathrm{mmol} / \mathrm{L}$ & $4.6 \pm 1.7$ & $4.6 \pm 1.1$ & $4.6 \pm 1.1$ & $4.6 \pm 1.1$ \\
\hline HDL-C, mmol/L & $1.3 \pm 0.4$ & $1.0 \pm 0.5^{\mathrm{b}}$ & $1.0 \pm 0.3^{b}$ & $1.2 \pm 0.9$ \\
\hline Hypertension, \% & 19.2 & $69.7^{\mathrm{b}}$ & $69.0^{\mathrm{b}}$ & $71.6^{\mathrm{b}}$ \\
\hline Diabetes, \% & 3.2 & $18.0^{\mathrm{b}}$ & $19.8^{b}$ & $12.8^{b}$ \\
\hline Hyperlipidemia, \% & 21.0 & $35.0^{\mathrm{b}}$ & $35.5^{\mathrm{b}}$ & $33.8^{\mathrm{b}}$ \\
\hline Smoking, \% & 37.3 & $46.9^{b}$ & $45.8^{b}$ & $50.0^{\mathrm{b}}$ \\
\hline
\end{tabular}

n, number of individuals; BMI, body mass index; SBP, systolic blood pressure; DBP, diastolic blood pressure; TCH, total cholesterol; HDL-C, high-density lipoprotein cholesterol. ${ }^{\mathrm{b}} \mathrm{P}<0.05$ vs control.

Table 2. Allele distribution of each polymorphism.

\begin{tabular}{|c|c|c|c|c|c|c|}
\hline SNP ID & Function $(\mathrm{M}>\mathrm{m})$ & Gene & Position & Population & MAF & $P_{\text {allele }}$ \\
\hline \multirow[t]{4}{*}{ rs1799983 } & (D298E) G>T & eNOS & Chr7:150327044 & Control & 0.108 & \\
\hline & & & & Ischemic stroke & 0.119 & NS \\
\hline & & & & Atherothrombosis & 0.116 & NS \\
\hline & & & & Lacunar infarction & 0.128 & NS \\
\hline \multirow[t]{4}{*}{ rs2070744 } & intron $\mathrm{T}>\mathrm{C}$ & eNOS & Chr7:150321012 & Control & 0.104 & \\
\hline & & & & Ischemic stroke & 0.113 & NS \\
\hline & & & & Atherothrombosis & 0.120 & NS \\
\hline & & & & Lacunar infarction & 0.095 & NS \\
\hline \multirow[t]{4}{*}{ rs2297518 } & (L608S) G>A & iNOS & Chr17:23120724 & Control & 0.169 & \\
\hline & & & & Ischemic stroke & 0.151 & NS \\
\hline & & & & Atherothrombosis & 0.132 & 0.025 \\
\hline & & & & Lacunar infarction & 0.206 & NS \\
\hline \multirow[t]{4}{*}{ rs841 } & intron $\mathrm{G}>\mathrm{A}$ & $\mathrm{GCH} 1$ & Chr14:54380242 & Control & 0.311 & \\
\hline & & & & Ischemic stroke & 0.377 & $0.001^{b}$ \\
\hline & & & & Atherothrombosis & 0.367 & 0.009 \\
\hline & & & & Lacunar infarction & 0.405 & $0.002^{b}$ \\
\hline \multirow[t]{4}{*}{ rs1049255 } & 3'-UTR C>T & CYBA & Chr16:87237238 & Control & 0.428 & \\
\hline & & & & Ischemic stroke & 0.389 & NS \\
\hline & & & & Atherothrombosis & 0.399 & NS \\
\hline & & & & Lacunar infarction & 0.361 & 0.038 \\
\hline \multirow[t]{4}{*}{ rs4673 } & $(\mathrm{Y} 72 \mathrm{H}) \mathrm{G}>\mathrm{A}$ & CYBA & Chr16:87240737 & Control & 0.078 & \\
\hline & & & & Ischemic stroke & 0.064 & NS \\
\hline & & & & Atherothrombosis & 0.068 & NS \\
\hline & & & & Lacunar infarction & 0.051 & 0.012 \\
\hline
\end{tabular}

MAF, minor allele frequency; $P_{\text {allele, }}$ value of allele was determined by $x^{2}$ test; NS, not significant. ${ }^{\mathrm{b}} \mathrm{FDR} q$ value $<0.05$.

association analysis assuming 3 common genetic models (codominant, dominant and recessive). Interestingly, SNP rs841 showed consistent effects using both a co-dominant (without adjustment for covariates, $P=0.001, q=0.009$; after adjustment 
for traditional risk factors, $P=0.003, q=0.027$ ) and a dominant model (without adjustment for covariates, $P=0.0003, q=0.0054$; after adjustment for traditional risk factors, $P=0.0006$, $q=0.0108$ ) (Table 3).

To test the possible effect of the IS subtypes in detecting an association, we then re-assessed the association between rs841 and the cerebral thrombosis and lacunar infarction groups (Supplementary Tables 1 and 2). It is of interest to note that rs841 was significantly associated with both subtypes (nominal $P<0.05)$. However, none of these results pass the significance threshold after multiple corrections. These negative results could be due to reduced sample size and statistical power for subtype analysis.

\section{Association of genotype combinations with ischemic stroke}

We applied the logistic regression analyses to test potential interactions among polymorphisms within genes involved in the NO-forming pathway that may confer IS risk and identified significant interactions between rs1049255 and rs841 $(P<0.001)$. In comparison with the reference combination of rs1049255 CC+CT and rs841 GG wild type genotypes, the combination of the rs1049255 CC+CT genotype together with the rs841 GA+AA genotype was found to be significantly associated with IS $(P=0.002, q=0.008$; Table 4$)$. The distribution combinations of other genotypes did not differ from the wildtype reference in overall IS. The combination of rs1049255 CC+CT and rs841 GA+AA genotypes was associated with significantly higher risk of IS even after adjustment for sex, age, and multiple cardiovascular risk factors (OR=1.73, 95\% CI: $1.27-2.35 ; P<0.0001, q<0.0001$ ) (Table 5). Given that the reduced sample size for IS subtypes resulted in largely insufficient power to test potential interactions, subtype analyses were not performed. Taken together, our data suggest an interaction of NO-forming pathway genes in the risk of IS.

\section{Discussion}

This study is to investigate the association between genetic polymorphisms in the genes implicated in NO production and risk for IS development in the Chinese Han population. The present results indicate that polymorphisms in GCH1 (rs841) are independently associated with an increased risk for IS. In contrast, we failed to detect significant independent association with the rest of SNPs even though they have been suggested to be associated with cardiovascular diseases such as hypertension, coronary heart disease or stroke ${ }^{[21-23]}$.

Nitric oxide, produced by nitric oxide synthase, is an important bioregulatory molecule and displays diverse biological activities. Tetrahydrobiopterin (BH4) is an essential cofactor for all three NOS isoforms, and basal enzyme activity correlates with the amount of $\mathrm{BH} 4$ bound tightly to $\mathrm{NOS}^{[24]}$. $\mathrm{BH} 4$ deficiency is proposed to lead to NOS uncoupling associated with decreased NO bioavailability and increased production of superoxide radicals from the uncoupled enzymatic form ${ }^{[25]}$. GTP cyclohydrolase 1 (GCH1) is the first-step and rate-limiting enzyme for $\mathrm{BH} 4$ biosynthesis in its de novo pathway ${ }^{[26]}$. Experi- mental mouse models with alterations in systemic or vascularspecific GCH1 expression have shown that GCH1 is a key regulator of vascular $\mathrm{BH} 4$ levels in vivo ${ }^{[27,28]}$. Low brain levels of $\mathrm{BH} 4$ have been shown in the mouse model for dominantly inherited GCH1 deficiency ${ }^{[29]}$. Recent evidence suggests that one SNP (rs841), located in the 3'-UTR of the GCH1 gene, is also associated with reduced biopterin-dependent effects ${ }^{[17]}$. The CYBA gene, located on the long arm of chromosome 16 at position 24, encodes human p22phox, which is an essential subunit for the functionality of the NADPH oxidase ${ }^{[30]}$. NADPH oxidase is an important enzymatic source of oxidative stress as well as uncoupled NOS caused by BH4 deficiency and plays a key role in the pathophysiology of several major cardiovascular diseases, including stroke ${ }^{[31]}$. Previous studies have shown the association between CYBA polymorphisms and vascular diseases $^{[30]}$. Our combined analysis identified GCH1 (rs841) and CYBA (rs1049255) interactions, indicating that these two functional polymorphisms may confer risk for IS through biological interactions with each other. Further studies might be required to ascertain whether CYBA (rs1049255) affects GCH1 expression and how such an effect might be mediated.

To control for potential false-positive results, we took several factors into consideration and carefully designed our study. First, all selected candidate SNPs have substantial functional effects that are likely involved in the development of IS. Second, assuming disease prevalence between $0.5 \%$ and $1 \%$, our combined sample size can reach $>98 \%$ power to detect a susceptibility locus with a genotypic relative risk $>1.65$ at the nominal type I error rate of $<0.05$ for SNPs with minor allele frequencies $>0.31$ under the dominant model. Third, we recruited only ethnically and geographically matched subjects from Chinese Han cohorts. Given the homogenous study population, we expect population substructure to be minimal. However, additional replication of the association signals in other independent cohorts is warranted.

In summary, genetic variants in the genes implicated in NO formation could have potentially important effects on the pathogenesis of vascular diseases, and genotyping of these variants may provide an additional tool to predict the risk for ischemic stroke in the Chinese Han population.

\section{Acknowledgements}

This study was supported by China 973 program (No 2007CB512004), and 863 program (№ 2006AA02A406).

\section{Author contribution}

Jiang-tao YAN, Lan ZHANG and Yu-jun XU performed the research; Xiao-jing WANG and Cong-yi WANG contributed new analytical tools and reagents; Jiang-tao YAN and Lan ZHANG analyzed the data; and Jiang-tao YAN, Lan ZHANG and Dao-wen WANG wrote the paper.

\section{Supplementary information}

Supplementary tables are available at Acta Pharmacologica Sinica website of NPG. 
Table 3. Genotype distribution in three models in ischemic stroke.

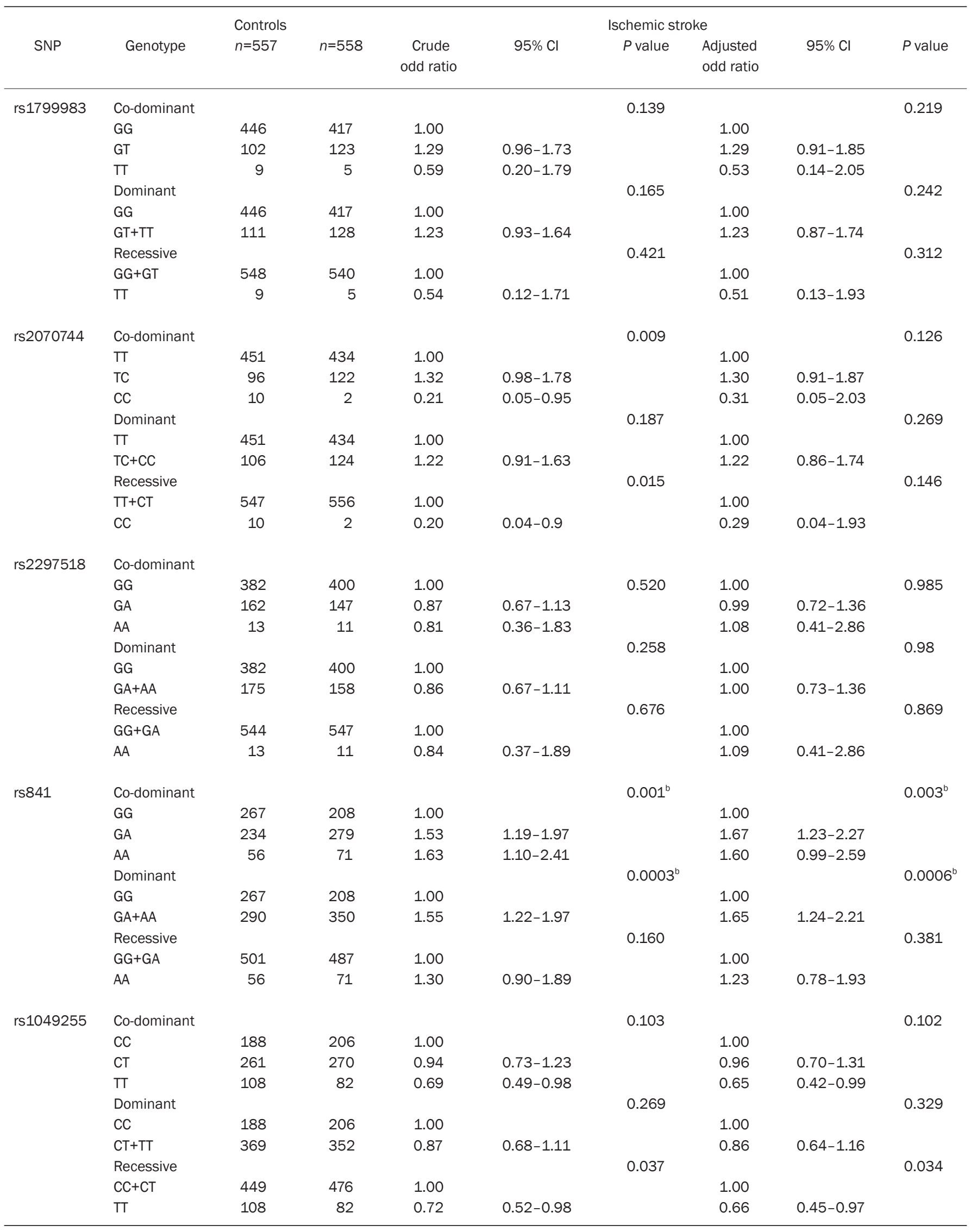




\begin{tabular}{|c|c|c|c|c|c|c|c|c|c|}
\hline \multirow[b]{2}{*}{ SNP } & \multirow[b]{2}{*}{ Genotype } & \multicolumn{2}{|l|}{ Controls } & \multirow[b]{2}{*}{$\begin{array}{c}\text { Crude } \\
\text { odd ratio }\end{array}$} & \multicolumn{4}{|c|}{ Ischemic stroke } & \multirow[b]{2}{*}{$P$ value } \\
\hline & & $n=557$ & $n=558$ & & $95 \% \mathrm{Cl}$ & $P$ value & $\begin{array}{l}\text { Adjusted } \\
\text { odd ratio }\end{array}$ & $95 \% \mathrm{Cl}$ & \\
\hline \multirow[t]{8}{*}{ rs4673 } & Co-dominant & & & & & 0.211 & & & 0.37 \\
\hline & $\mathrm{GG}$ & 471 & 487 & 1.00 & & & 1.00 & & \\
\hline & GA & 85 & 71 & 0.81 & $0.58-1.13$ & & 0.77 & $0.50-1.16$ & \\
\hline & GG & 471 & 487 & 1.00 & & & 1.00 & $0.50-1.16$ & \\
\hline & $\mathrm{GA}+\mathrm{AA}$ & 86 & 71 & 0.80 & $0.57-1.12$ & & 0.76 & & \\
\hline & Recessive & & & & & 0.499 & & & 0.516 \\
\hline & $\mathrm{GG}+\mathrm{GA}$ & 556 & 558 & 1.00 & & & 1.00 & & \\
\hline & $\mathrm{AA}$ & 1 & 0 & / & / & & / & / & \\
\hline
\end{tabular}

$\mathrm{Cl}$, confidence interval; SNP, single-nucleotide polymorphism. Adjusted odd ratios were adjusted for gender, age, body mass index, hypertension, diabetes, hyperlipidemia and smoking status. ${ }^{\mathrm{b}} \mathrm{FDR} q$ value $<0.05$.

Table 4. Distribution of combined genotypes in overall ischemic stroke.

\begin{tabular}{llccl}
\hline \multicolumn{1}{c}{ Genotype } & $\begin{array}{c}\text { Ischemic } \\
\text { stroke } \\
\text { rs1049255 }\end{array}$ & rs841 & $\begin{array}{c}\text { Control } \\
(n=558)\end{array}$ & $P$ value \\
$(n=557)$ & \\
\hline CC+CT & GG & 175 & 210 & reference \\
CC+CT & GA+AA & 301 & 239 & 0.002 \\
TT & GG & 33 & 57 & 0.130 \\
TT & GA+AA & 49 & 51 & 0.526 \\
\hline
\end{tabular}

Table 5. Analysis of multiple logistic regression model for synergism genotypes in ischemic stroke group.

\begin{tabular}{lccrc}
\hline \multirow{2}{*}{ Variable } & \multirow{2}{*}{ Adjusted OR } & \multicolumn{2}{c}{$95 \% \mathrm{Cl}$} & \multirow{2}{*}{ P value } \\
& & Lower & Upper & \\
\hline Gender $(\mathrm{m} / \mathrm{f})$ & 0.58 & 0.39 & 0.87 & 0.01 \\
Age $(n)$ & 0.96 & 0.95 & 0.98 & $<0.0001$ \\
Hypertension $(\mathrm{y} / \mathrm{n})$ & 7.20 & 5.11 & 10.16 & $<0.0001$ \\
Diabetes $(\mathrm{y} / \mathrm{n})$ & 3.36 & 1.79 & 6.32 & $<0.0001$ \\
Hyperlipidemia $(\mathrm{y} / \mathrm{n})$ & 1.48 & 1.02 & 2.15 & 0.04 \\
Smoking $(\mathrm{y} / \mathrm{n})$ & 2.18 & 1.49 & 3.18 & $<0.0001$ \\
rs1049255(CC+CT)* & 1.73 & 1.27 & 2.35 & $<0.0001$ \\
rs841(GA+AA) & & & & \\
\hline
\end{tabular}

OR, odd ratio; Variables included in the model were gender, age, body mass index, hypertension, diabetes, hyperlipidemia and smoking status. Nonsignificant variables $(P>0.05)$ have not been shown in the table.

\section{References}

1 Jia Q, Liu LP, Wang YJ. Stroke in China. Clin Exp Pharmacol Physiol 2010; 37: 259-64.

2 Meschia JF, Brott TG, Brown RD Jr, Crook RJ, Frankel M, Hardy J, et al. The ischemic stroke genetics study (ISGS) protocol. BMC Neurol 2003; 3: 4.

3 ladecola C, Pelligrino DA, Moskowitz MA, Lassen NA. Nitric oxide synthase inhibition and cerebrovascular regulation. J Cereb Blood Flow
Metab 1994; 14: 175-92.

4 Faraci FM, Brian JE Jr. Nitric oxide and the cerebral circulation. Stroke 1994; 25: 692-703.

5 Forman $\sqcup$, Liu P, Nagele RG, Yin K, Wong PY. Augmentation of nitric oxide, superoxide, and peroxynitrite production during cerebral ischemia and reperfusion in the rat. Neurochem Res 1998; 23: 141-8.

6 Yogo K, Shimokawa H, Funakoshi H, Kandabashi T, Miyata K, Okamoto $\mathrm{S}$, et al. Different vasculoprotective roles of NO synthase isoforms in vascular lesion formation in mice. Arterioscler Thromb Vasc Biol 2000; 20: E96-E100.

7 Wang SS, Davis S, Cerhan JR, Hartge P, Severson RK, Cozen W, et al. Polymorphisms in oxidative stress genes and risk for non-Hodgkin lymphoma. Carcinogenesis 2006; 27: 1828-34.

8 Alvarez R, Gonzalez P, Batalla A, Reguero JR, Iglesias-Cubero G, Hevia $\mathrm{S}$, et al. Association between the NOS3 (-786 T/C) and the ACE (I/D) DNA genotypes and early coronary artery disease. Nitric Oxide 2001; 5: 343-8.

9 Shimasaki Y, Yasue H, Yoshimura M, Nakayama M, Kugiyama K, Ogawa $\mathrm{H}$, et al. Association of the missense Glu298Asp variant of the endothelial nitric oxide synthase gene with myocardial infarction. J Am Coll Cardiol 1998; 31: 1506-10.

10 Hingorani AD, Liang CF, Fatibene J, Lyon A, Monteith S, Parsons A, et al. A common variant of the endothelial nitric oxide synthase (Glu298 $\rightarrow$ Asp) is a major risk factor for coronary artery disease in the UK. Circulation 1999; 100: 1515-20.

11 Cai H, Wilcken DE, Wang XL. The Glu-298 $\rightarrow$ Asp $(894 G \rightarrow T)$ mutation at exon 7 of the endothelial nitric oxide synthase gene and coronary artery disease. J Mol Med 1999; 77: 511-4.

12 Nakayama M, Yasue H, Yoshimura M, Shimasaki Y, Kugiyama K, Ogawa $\mathrm{H}$, et al. T-786 $\rightarrow \mathrm{C}$ mutation in the 5 '-flanking region of the endothelial nitric oxide synthase gene is associated with coronary spasm. Circulation 1999; 99: 2864-70.

13 Guzik TJ, West NE, Black E, McDonald D, Ratnatunga C, Pillai R, et al. Functional effect of the C242T polymorphism in the NAD(P)H oxidase p22phox gene on vascular superoxide production in atherosclerosis. Circulation 2000; 102: 1744-7.

14 Inoue N, Kawashima S, Kanazawa K, Yamada S, Akita H, Yokoyama M. Polymorphism of the NADH/NADPH oxidase p22 phox gene in patients with coronary artery disease. Circulation 1998; 97: 135-7.

15 Li A, Prasad A, Mincemoyer R, Satorius C, Epstein N, Finkel T, et al. Relationship of the C242T p22phox gene polymorphism to angio- 
graphic coronary artery disease and endothelial function. Am J Med Genet 1999; 86: 57-61.

16 Cahilly C, Ballantyne CM, Lim DS, Gotto A, Marian AJ. A variant of p22(phox), involved in generation of reactive oxygen species in the vessel wall, is associated with progression of coronary atherosclerosis. Circ Res 2000; 86: 391-5.

17 Zhang L, Rao F, Zhang K, Khandrika S, Das M, Vaingankar SM, et al. Discovery of common human genetic variants of GTP cyclohydrolase 1 (GCH1) governing nitric oxide, autonomic activity, and cardiovascular risk. J Clin Invest 2007; 117: 2658-71.

18 Wang Q, Ding H, Tang JR, Zhang L, Xu YJ, Yan JT, et al. C-reactive protein polymorphisms and genetic susceptibility to ischemic stroke and hemorrhagic stroke in the Chinese Han population. Acta Pharmacol Sin 2009; 30: 291-8.

19 Storey JD, Tibshirani R. Statistical significance for genomewide studies. Proc Natl Acad Sci U S A 2003; 100: 9440-5.

20 QUANTO 1.1: A computer program for power and sample size calculations for genetic-epidemiology studies, http://hydra.usc.edu/gxe; 2006.

21 Hassan A, Gormley K, O'Sullivan M, Knight J, Sham P, Vallance P, et al. Endothelial nitric oxide gene haplotypes and risk of cerebral smallvessel disease. Stroke 2004; 35: 654-9.

22 Szolnoki Z, Havasi V, Bene J, Komlosi K, Szoke D, Somogyvari F, et al. Endothelial nitric oxide synthase gene interactions and the risk of ischaemic stroke. Acta Neurol Scand 2005; 111: 29-33.

23 Markus HS, Ruigrok Y, Ali N, Powell JF. Endothelial nitric oxide syn- thase exon 7 polymorphism, ischemic cerebrovascular disease, and carotid atheroma. Stroke 1998; 29: 1908-11.

24 Moens AL, Kass DA. Tetrahydrobiopterin and cardiovascular disease. Arterioscler Thromb Vasc Biol 2006; 26: 2439-44.

25 Vasquez-Vivar J, Kalyanaraman B, Martasek P, Hogg N, Masters BS, Karoui $\mathrm{H}$, et al. Superoxide generation by endothelial nitric oxide synthase: the influence of cofactors. Proc Natl Acad Sci U S A 1998; 95: 9220-5.

26 Auerbach G, Nar H. The pathway from GTP to tetrahydrobiopterin: three dimensional structures of GTP cyclohydrolase I and 6-pyruvoyl tetrahydropterin synthase. Biol Chem 1997; 378: 185-92.

27 Khoo JP, Nicoli T, Alp NJ, Fullerton J, Flint J, Channon KM. Congenic mapping and genotyping of the tetrahydrobiopterin-deficient $\mathrm{hph}-1$ mouse. Mol Genet Metab 2004; 82: 251-4.

28 Khoo JP, Zhao L, Alp NJ, Bendall JK, Nicoli T, Rockett K, et al. Pivotal role for endothelial tetrahydrobiopterin in pulmonary hypertension. Circulation 2005; 111: 2126-33.

29 Hyland K, Gunasekara RS, Munk-Martin TL, Arnold LA, Engle T. The hph-1 mouse: a model for dominantly inherited GTP-cyclohydrolase deficiency. Ann Neurol 2003; 54: S46-8.

30 San Jose G, Fortuno A, Beloqui O, Diez J, Zalba G. NADPH oxidase CYBA polymorphisms, oxidative stress and cardiovascular diseases. Clin Sci (Lond) 2008; 114: 173-82.

31 Mueller CF, Laude K, McNally JS, Harrison DG. ATVB in focus: redox mechanisms in blood vessels. Arterioscler Thromb Vasc Biol 2005; 25: $274-8$. 